\title{
Coalescence-induced jumping of immersed and suspended droplets on microstructured substrates
}

\author{
Samaneh Farokhirad ${ }^{a}$, Mahmood Mohammadi Shad ${ }^{\mathrm{b}}$ and Taehun Lee ${ }^{\mathrm{b}}$ \\ ${ }^{a}$ Department of Chemical and Biomolecular Engineering, University of Pennsylvania, Philadelphia, PA, \\ USA; ${ }^{b}$ Department of Mechanical Engineering, City College of City University of New York, New York, \\ NY, USA
}

\begin{abstract}
The coalescence-induced jumping of liquid droplets on superhydrophobic structured substrates is investigated numerically using a three-dimensional multiphase lattice Boltzmann method. The numerical experiments on evolution of droplets during jumping process show higher jumping velocity and height from superhydrophobic substrates structured with a periodic array of square pillars, than flat superhydrophobic substrates with an equilibrium contact angle of $180^{\circ}$. The results further reveal a strong effect of pillars on the vertical jumping velocity and the final quasiequilibrium height of the merged droplet as a function of air and liquid viscosity, as well as air inertia. As for substrate wettability, it is found that, compared to the flat superhydrophobic substrate, the critical contact angle where the merged droplet jumps away from substrate is reduced for pillared substrate and is about $120^{\circ}$. It is also observed that the droplet initial placement on a substrate with a square array of pillars has an important effect on the spontaneous jumping of the coalesced droplet, and a Wenzel-Cassie wetting transition upon coalescence is observed for droplets that are initially immersed within the pillars.
\end{abstract}

\section{ARTICLE HISTORY}

Received 23 September 2016

Accepted 30 October

2016

\section{KEYWORDS}

Lattice Boltzmann method; droplet coalescence; structured superhydrophobic substrate; self-propelled jumping; Wenzel-Cassie wetting

\section{Introduction}

Quite recently, considerable attention has been paid to spontaneous motion of droplets from superhydrophobic substrates due to its importance in various biological and industrial applications such as self-cleaning surfaces, phase change heat exchangers and anti-icing. The focus of recent studies has been on the rapid removal of dropwise condensation from non-wetting substrate to improve the phase change heat transfer performance (Chen et al., 2007; Chen et al., 2011; Feng et al., 2012; Miljkovic, Enright, \& Wang, 2012). Coalescence-induced selfpropelled jumping of condensed droplets is a good candidate for an efficient condensation mechanism in the phase change heat exchangers where no external force is preferred. In this newly discovered physical mechanism, which was first reported by Boreyko and Chen (2009), the superhydrophobic nature of substrates 
help the condensed droplets approach each other, merge upon coalescence and eventually jump away from the substrate due to energy conversion from surface energy to the kinetic energy. This enhanced self-propelled jumping would be promising for frost layer removal from industrial facilities such as heat exchangers suffering from frost formation in cold winter. Recently, an effective superhydrophobic aluminium substrate was fabricated that can slow the growth rate of frost formation and delay its propagation more than 4 times than that on a smooth aluminium substrate (Kim, Lee, Kim, \& Kim, 2015). However, the self-propelled jumping behaviour of droplets has not been observed for droplet sizes comparable to or larger than the capillary length, where gravitational force becomes effective. Furthermore, such non-wetting substrates presenting self-propelled jumping of droplets were shown insensitive to the contact angle hysteresis (less than 5\%) (Miljkovic, Enright, \& Wang, 2013), which is due to the spontaneous removal of the merged droplet at length scales well below the capillary length.

Numerous studies have presented both quantitative and semi-quantitative investigations of this new physical mechanism of droplet removal from superhydrophobic substrates, which are either flat or geometrically rough using experimental, theoretical and numerical methods (Chen, Patel, Weibel, \& Garimella, 2016; Liu \& Cheng, 2015; Farokhirad, Morris, \& Lee, 2015; Liu, Ghigliotti, Feng, \& Chen, 2014; Liu, Sun, Sun, \& Ai, 2012; Miljkovic et al., 2013; Nam, Kim \& Shin, 2013; Peng et al., 2013; Shi, Tang, \& Xia, 2015; Wang, Yang, \& Zhao, 2011; Wisdom, Watson, Liu, Watson, \& Chen, 2013). However, all physical behaviours of this autonomous motion reported on flat superhydrophobic substrates are also dependent on the shape and structure of the substrate which can be heterogeneous or pillared in real applications. To the authors' best knowledge, few numerical studies can be found in the literature addressing the self-propelled jumping of droplets on microstructured substrates (Liu \& Cheng, 2015; Shi et al., 2015).

Liu and Cheng (2015) adopted a three-dimensional lattice Boltzmann method (LBM) based on the pseudo-potential multiphase LBM, to study the effects of periodic pillar arrays on the self-propelled jumping of droplets. It was concluded that depending on the size of the droplets, there exists an optimal spacing between the consecutive pillars where the maximum jumping height can be achieved.

Shi et al. (2015) simulated self-propelled jumping of the coalesced droplet on flat substrates covered with conical posts using three-dimensional multiple relaxation time pseudo-potential LBM. They focused on the effects of the height and the spacing of the substrate's conical posts, as well as the substrate wettability, on the jumping behaviour of droplet. The numerical results showed that with increasing the height of the conical posts and decreasing the spacing between them, the merged droplet reaches a higher distance from the substrate. Their results also demonstrated that the jumping height of the merged droplet can be 
enhanced further by increasing the contact angle between the textured substrate and the droplet.

The objective of this study is to investigate the effect of the ambient environment on the jumping removal process of droplets on microstructured superhydrophobic substrates using a recently developed multiphase LBM (Lee, 2009; Lee \& Liu, 2010). The implemented LBM method is based on the CahnHilliard diffuse interface theory for binary fluids and is capable of eliminating parasitic currents at equilibrium, thus can employ components with sufficiently large density differences (Zhang, Farokhirad, Lee, \& Koplik, 2014). A recent study of coalescence-induced jumping of droplet on flat superhydrophobic substrates revealed that the inertia of surrounding air has no dynamic role prior to jumping of the merged droplet (Farokhirad et al., 2015). However, the air inertia was shown to play a key role at later stages of the jumping process where the merged droplet has already left the substrate (Farokhirad et al., 2015). Moreover, a liquid droplet on a structured superhydrophobic substrate can exhibit either a suspended or an immersed state. It was also observed that both suspended and immersed wetting regimes can coexist on the same substrate (Forsberg, Nikolajeff, \& Karlsson, 2011). In the current study, it will be shown that the selfpropulsion mechanism can be employed to promote rapid removal of droplets trapped in the immersed state.

The rest of this paper is organised as follows. Firstly, we briefly review the implementation of the multiphase LBM used in our simulations in Section 2. Secondly, numerical results for jumping of droplets on microstructured superhydrophobic substrates are discussed in Section 3. Finally, a brief conclusion is drawn in Section 4.

\section{LBM for binary fluids}

In our numerical simulations, we implement the LBM for binary immiscible fluids developed by Lee (2009) and Lee and Liu (2010), which is based on the Cahn-Hilliard diffuse interface theory. In the Cahn-Hilliard equation (Cahn, 1977), the interface between binary fluids evolves so that the total free energy of the system $(\Psi)$ is minimised. The total free energy encompasses both local and non-local terms $\left(\Psi_{b}\right)$, and a surface term $\left(\Psi_{s}\right)$, which controls the interaction between the solid surface and the interface of binary fluids, and is written as follows:

$$
\begin{aligned}
\Psi=\Psi_{b}+\Psi_{s}= & \int_{V}\left[E_{0}(C)+\frac{\kappa}{2}|\nabla C|^{2}\right] \mathrm{d} V \\
& +\int_{S}\left[\phi_{0}-\phi_{1} C_{s}+\phi_{2} C_{s}^{2}-\phi_{3} C_{s}^{3}+\ldots\right] \mathrm{d} S,
\end{aligned}
$$

where $V$ is the volume of the system and $S$ is the surface area of the substrate. The local part of the bulk free energy, namely the mixing energy, is $E_{0}(C)=$ $\beta C^{2}(C-1)^{2}$, where $\beta$ is a constant, and $C$ is the composition or the order 
parameter, indicating the local concentration of each species. The coefficient $\kappa$ in the non-local term controls the surface tension and interfacial thickness. In the surface integral, $C_{s}$ denotes the composition at the solid surface and the $\phi_{i}$ 's $(i=0, \ldots, 3)$ are constant coefficients. Given the composition, the mixture density of the system is obtained by $\rho=C \rho_{1}+(1-C) \rho_{2}$, where $\rho_{1}$ and $\rho_{2}$ are the bulk densities of the two fluids. The integral of the free energy on solid boundaries $\left(\Psi_{s}\right)$ employs a cubic boundary condition (Liu \& Lee, 2009) which specifies the parameter sets: $\phi_{0}=\phi_{1}=0, \phi_{2}=\phi_{c} / 2$, and $\phi_{3}=\phi_{c} / 3$, where $\phi_{c}$ is chosen to recover the desired contact angle at equilibrium.

The out of equilibrium binary system advances towards its minimum energy configuration according to the Cahn-Hilliard equation:

$$
\partial_{t} C+\nabla \cdot(\mathbf{u} C)=\nabla \cdot(M \nabla \mu)
$$

where $\mathbf{u}$ is the volume-averaged mixture velocity and $M$ is the mobility parameter. The chemical potential $\mu$ is obtained from the total free energy, upon variation (Cahn, 1977) and is written as $\mu=\mu_{0}-\kappa \nabla^{2} C$, with $\mu_{0}=\partial E_{0} / \partial C$ being the classical part of the chemical potential.

The solution to Equation (2) requires two boundary conditions. The boundary condition for $\nabla^{2} \mu$ ensures no mass flux due to the non-zero chemical potential gradient in the direction normal to the solid surface $\left(\left.\mathbf{n} \cdot \nabla \mu\right|_{s}=0\right)$, where $\mathbf{n}$ is the normal unit vector to the surface. The boundary condition for $\nabla^{2} C$, is established by minimising the surface free energy subject to the specified wall free energy (Liu \& Lee, 2009). As mentioned, this study uses a cubic boundary condition at equilibrium. Therefore, the boundary condition for $\nabla^{2} C$ becomes $\left.\mathbf{n} \cdot \nabla C\right|_{s}=\frac{\phi_{c}}{\kappa}\left(C_{s}-C_{s}^{2}\right)$. The equilibrium profile for a planar interface is then obtained by minimising the local term of Equation 1 and is given by $C(z)=$ $.5+.5 \tanh (2 z / \zeta)$, where $z$ is the coordinate normal to the interface and $\zeta$ is the interface thickness. Given the surface tension and interface thickness, $\beta$ and $\kappa$ can be specified as $\beta=12 \sigma / \zeta$ and $\kappa=\beta \zeta^{2} / 8$, respectively.

In LBM, one follows the evolution of a density probability distribution function for fictitious particles moving on a lattice. Two particle distribution functions, $g_{\alpha}$ and $h_{\alpha}$, are used for binary fluids (Lee \& Liu, 2010). The former is used for the calculation of pressure and momentum of a two-component mixture, and the latter is used as a phase field function for the transport of order parameter. The discrete Boltzmann equations for pressure evolution and momentum equations, and advective phase field equation (Lee \& Liu, 2010) are, respectively:

$$
\begin{aligned}
\frac{\partial g_{\alpha}}{\partial t}+\mathbf{e}_{\alpha} \cdot \nabla g_{\alpha}= & -\frac{1}{\lambda}\left(g_{\alpha}-g_{\alpha}^{e q}\right) \\
& +\left(\mathbf{e}_{\alpha}-\mathbf{u}\right) \cdot\left[\nabla \rho c_{s}^{2}\left(\Gamma_{\alpha}-\Gamma_{\alpha}(0)\right)+\mu \nabla C \Gamma_{\alpha}\right], \\
\frac{\partial h_{\alpha}}{\partial t}+\mathbf{e}_{\alpha} \cdot \nabla h_{\alpha}= & -\frac{1}{\lambda}\left(h_{\alpha}-h_{\alpha}^{e q}\right)+\nabla \cdot(M \nabla \mu) \Gamma_{\alpha}
\end{aligned}
$$




$$
+\left(\mathbf{e}_{\alpha}-\mathbf{u}\right) \cdot\left[\nabla C-\frac{C}{\rho c_{s}^{2}}(\nabla p+\mu \nabla C)\right] \Gamma_{\alpha},
$$

where $\mathbf{e}_{\alpha}$ is the discretised velocity, $c_{s}$ is the speed of sound, $\lambda$ is the relaxation time and $h_{\alpha}^{e q}$, and $g_{\alpha}^{e q}$ are the equilibrium distribution functions given by:

$$
\begin{aligned}
& g_{\alpha}^{e q}=t_{\alpha} p+t_{\alpha} \rho c_{s}^{2}\left(\frac{\mathbf{e}_{\alpha} \cdot \mathbf{u}}{c_{s}^{2}}+\frac{\left(\mathbf{e}_{\alpha} \cdot \mathbf{u}\right)^{2}}{2 c_{s}^{4}}-\frac{\mathbf{u} \cdot \mathbf{u}}{2 c_{s}^{2}}\right), \\
& h_{\alpha}^{e q}=t_{\alpha} C\left(1+\frac{\mathbf{e}_{\alpha} \cdot \mathbf{u}}{c_{s}^{2}}+\frac{\left(\mathbf{e}_{\alpha} \cdot \mathbf{u}\right)^{2}}{2 c_{s}^{4}}-\frac{\mathbf{u} \cdot \mathbf{u}}{2 c_{s}^{2}}\right),
\end{aligned}
$$

where $t_{\alpha}$ is the weight corresponding to $\mathbf{e}_{\alpha}$, and $p$ is the pressure. The relaxation time $\tau=\lambda / \delta t$ is related to the kinematic viscosity by $v=\tau c_{s}^{2} \delta t$ and $\Gamma_{\alpha}$ is given as $\Gamma_{\alpha}=\Gamma_{\alpha}(\mathbf{u})=h_{\alpha}^{e q} / C$. The lattice Boltzmann equations (LBE) for Equations (3) and (4) are obtained by applying a trapezoidal approximation for integration along characteristics over time step $\delta t$ (Lee \& Liu, 2010). The macroscopic variables such as $C, \rho \mathbf{u}$, and $p$ are then computed from respective moments of the distribution functions.

\section{Results and discussion}

In this section, the LBM method described in Section 2 is implemented to simulate the coalescence-induced jumping of droplets on superhydrophobic structured substrates. Realistic solid surfaces are not often smooth and a variation in their roughness height is the general case. Here we consider a periodic array of square pillars, since such surfaces can exhibit superhydrophobic behaviour that are of special interest because of the interplay of suspended and immersed states (Wenzel, 1936).

The problem geometry is given in Figure 1, and it consists of square pillars of width $w$ and height $h$ arranged in a square array. Two spherical liquid droplets are initially placed very close to each other on the tip of square pillars. The simulation parameters that define this problem are the dynamic viscosity and density of the droplet $\left(\mu_{d}, \rho_{d}\right)$ and air $\left(\mu_{a}, \rho_{a}\right)$, respectively, the droplet-air surface tension $(\sigma)$, the droplet radius $\left(r_{d}\right)$ and the corresponding pillar geometries $(w, s, h)$. The dimensionless groups to describe the coalescence-induced jumping of droplets on a structured substrate are the droplet-air viscosity ratio $\left(\mu_{d} / \mu_{a}\right)$, droplet-air density ratio $\left(\rho_{d} / \rho_{a}\right)$ the Ohnesorge number $\left(O h=\mu_{d} / \sqrt{\rho_{d} \sigma r_{d}}\right)$, the contact angle between the droplet and the textured substrate $(\theta)$, and pillar-droplet size ratios $\left(w / r_{d}, h / r_{d}, s / r_{d}\right)$. The full set of simulation parameters that define the system are listed in Table 1.

We describe the dynamics of the merged droplet, by defining its instantaneous velocity as the $y$-component velocity of its mass centre: 


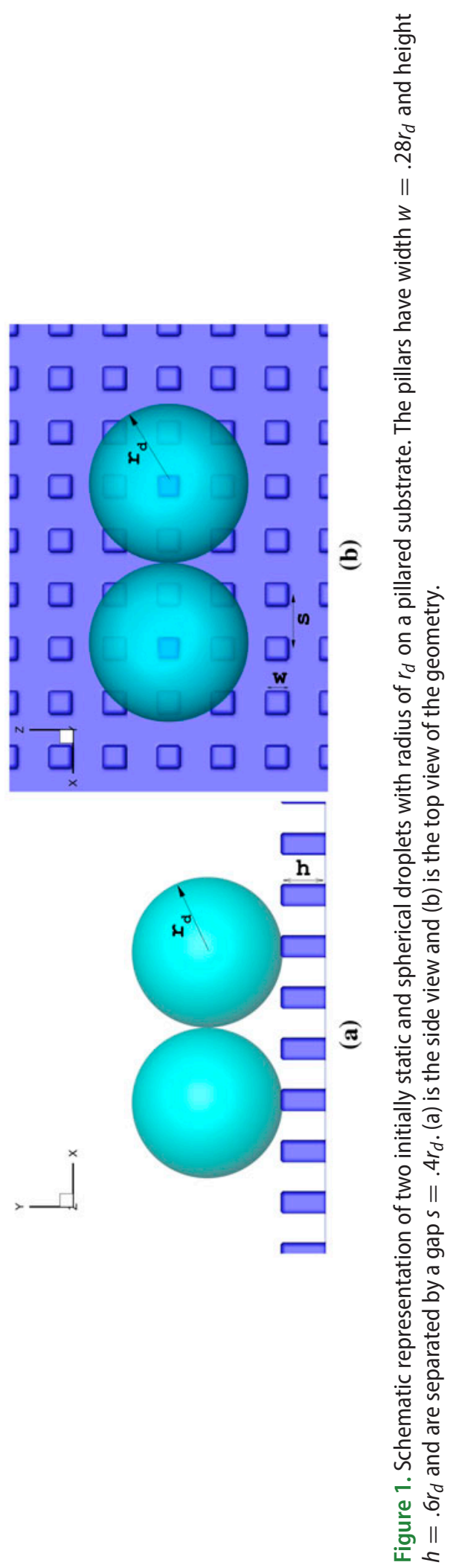


Table 1. The dimensionless simulation parameters for coalescence-induced jumping of droplet problem.

\begin{tabular}{lcc}
\hline Dimensionless parameters & Symbol & Value \\
\hline Droplet-Air density ratio & $\rho_{r}=\rho_{d} / \rho_{a}$ & $100-800$ \\
Droplet-Air viscosity ratio & $\mu_{r}=\mu_{d} / \mu_{a}$ & $15-60$ \\
Ohnesorge number & $\mathrm{Oh}$ & $.02-.4$ \\
Substrate equilibrium contact angle & $\theta$ & $110^{\circ}-160^{\circ}$ \\
Pillar width/Droplet radius & $w / r_{d}$ & .28 \\
Pillar height/Droplet radius & $h / r_{d}$ & .6 \\
Pillar separation/Droplet radius & $\mathrm{s} / r_{d}$ & .4 \\
\hline
\end{tabular}

$$
\bar{v}=\frac{\int_{V} C v_{y} d V}{\int_{V} C d V}
$$

where $y$ is the vertical direction normal to the substrate and $C$ localises the calculation to the droplet. The jumping time and velocity of the merged droplet are scaled by the characteristic merging time and velocity, respectively, and are given as follows:

$$
\begin{aligned}
\tau_{c i}=\sqrt{\frac{\rho_{d} r_{d}^{3}}{\sigma}}, \\
u_{c i}=\sqrt{\frac{\sigma}{\rho_{d} r_{d}}} .
\end{aligned}
$$

\subsection{Capillary-inertial jumping process of suspended droplets}

Figure 2(a) shows the evolution of two initially suspended droplets $\left(r_{d}=16 \mu \mathrm{m}\right.$ corresponding to 25 lattices) during coalescence and jumping processes on a superhydrophobic substrate structured with regularly spaced pillars. At the beginning of the evolution $\left(t^{*}=0\right)$ the coalescence is initiated due to interface overlapping of droplets with each other. Then a tiny liquid bridge is formed between coalescing droplets and expands rapidly due to interfacial tension of droplets.

The expanding bridge at $t^{*}=.96$ reaches the tip of the pillar structure and the merged droplet starts an upward motion and gradually reduces its contact area with the pillar surface around $t^{*}=1.44$. This contact area eventually becomes zero at the point of departure, when the bottom of the merged droplet leaves the tip of pillar structure $\left(t^{*}=2.88-4.80\right)$. The detached droplet experiences oscillations between the oblate and prolate shapes, while moving in upward direction and relaxes to a larger spherical shape, after several oscillations $\left(t^{*}=\right.$ $5.76-63.2)$.

In Figure 2(b), the capillary-inertial jumping process of droplet on a flat substrate (FS) with an equilibrium contact angle of $\theta=180^{\circ}$ is illustrated and is compared with that of droplets on a pillared substrate (PS), shown in Figure 2(a). The initial evolution including formation and expansion of liquid 
bridge occurs faster on a PS due to a smaller contact area between the droplets and the substrate. Therefore, compared to a FS, less amount of the released surface energy during coalescence on a PS is suppressed by the viscous dissipation energy. Self-propelled jumping occurs only when the excess amount of the released surface energy overcomes the adhesion of coalesced droplets to the substrate. In the case of a PS, the adhesion between the droplet and the pillars is reduced by introducing more droplet-air interface and the merged droplet is more likely to jump away directly and reach out to a higher distance from the PS.

Similar to the droplet removal upon coalescence on an FS (Liu et al., 2014), the jumping process for droplets on a PS is divided into four stages, as illustrated in Figure 3: (I) formation and expansion of the liquid bridge between the coalescing droplets; (II) acceleration of the merged droplet on the substrate towards its maximum velocity; (III) detachment of the merged droplet from the substrate; and (IV) reduction of the upward motion of droplet in the air due to the presence of air friction. The droplet eventually relaxes to a larger spherical shape, after several oscillations in stage IV.

To further illustrate the comparison of jumping processes shown in Figure 2, we plot the temporal evolution of the instantaneous droplet vertical velocity defined in Equation 7 in Figure 4. Based on this figure, the merged droplet on a PS attains a smaller maximum velocity prior to departure. The total droplet-air interfacial area in PS is smaller than FS which results in the release of less surface energy during coalescence. However, compared to FS, a small amount of the released surface energy in PS is surpassed by the energy consumed through the viscous dissipation prior to droplet departure from the substrate, which is due to the air entrapped among the gaps between pillars. Therefore, more energy is available for the self-propelled motion of droplet from the PS, and it leaves the PS sooner and attains a higher quasi-equilibrium jumping velocity.

It must be noted that the spacing between pillars on a structured superhydrophobic substrate can play a substantial role on the jumping of coalesced droplets. By increasing the spacing between the pillars, lower portion of the droplet can touch the bottom of the substrate, and as a result, the interaction time increases (Liu \& Cheng, 2015). Therefore, more of the released surface energy can be overcome by the viscous dissipation energy, so that the merged droplet attains its spherical shape without jumping from the structured substrate.

\subsubsection{Effect of density and viscosity ratios on the jumping process}

Effect of viscosity is illustrated by temporal evolution of the instantaneous droplet vertical velocity for different $O h$ in Figure 5. The $O h$ is varied from .0217 to .375 corresponding to the variation of droplet and air viscosities while keeping all other parameters including $\mu_{r}=12.9$ and $\rho_{r}=50$ fixed.

As $\mathrm{Oh}$ increases, the maximum jumping velocity in stage II decreases and the deceleration velocity during subsequent stages increases because of viscous effect. With $\mathrm{Oh}>.3$, the viscous effect becomes stronger and it dissipates the 

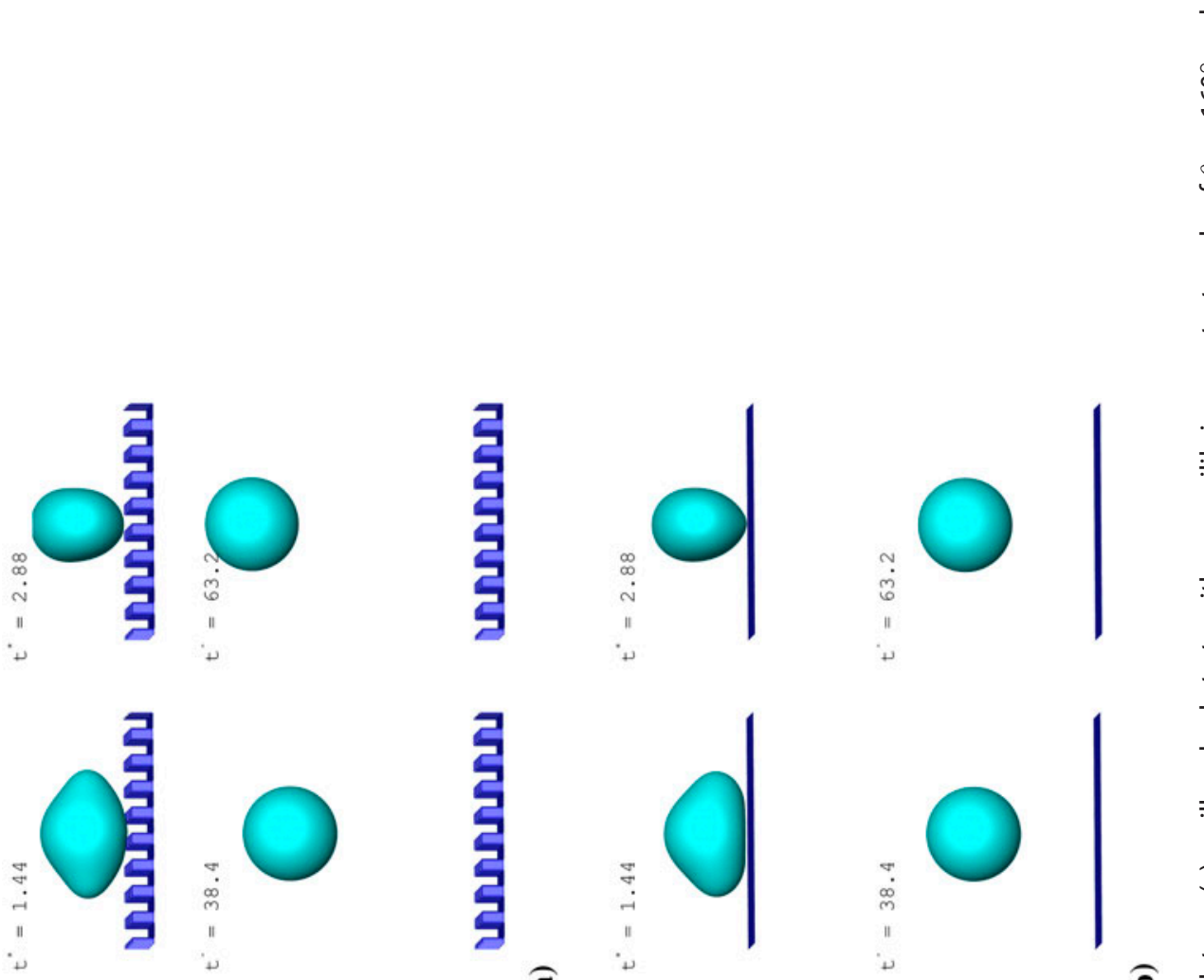

זั

:

II

$\stackrel{\oplus}{4}$
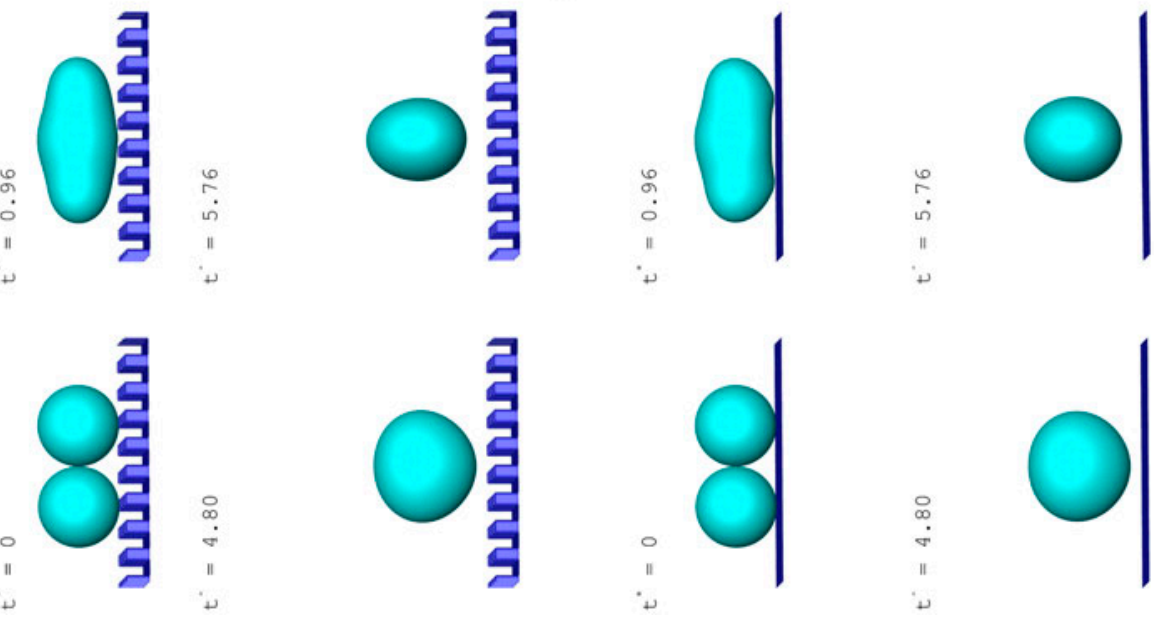

듬

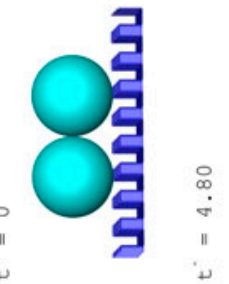

$\circ$
$\infty$
$\dot{\sigma}$
"
".

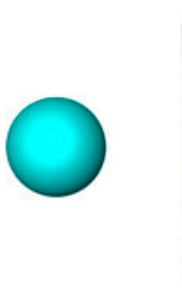




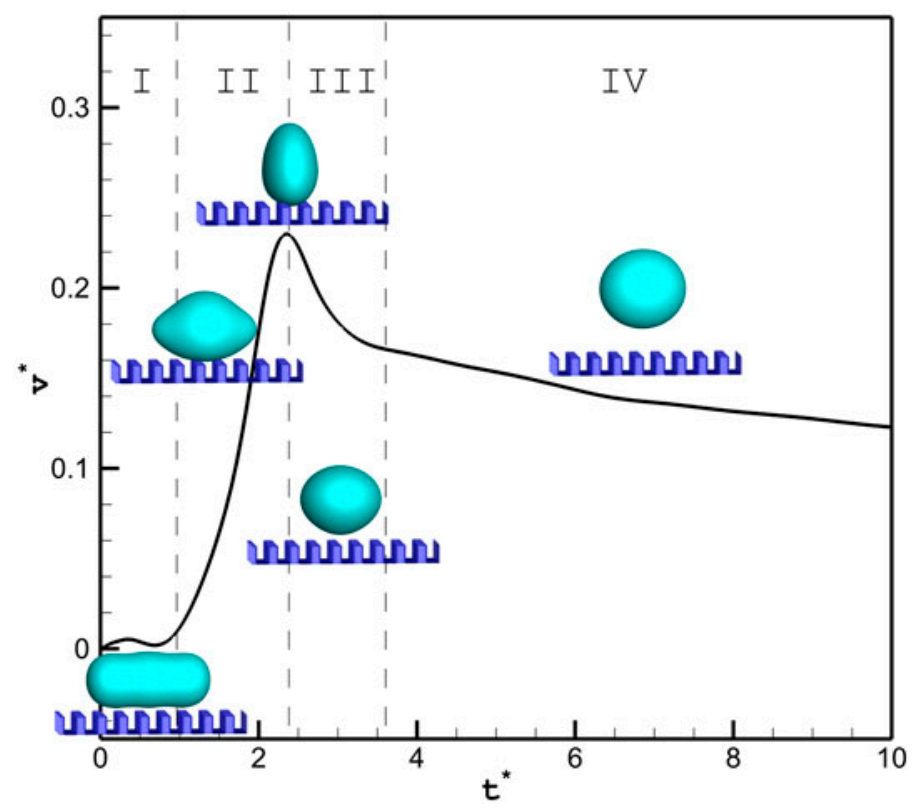

Figure 3. Temporal evolution of the instantaneous droplet vertical velocity during the jumping process illustrated in Figure 2(a).

Note: The velocity and time are represented as dimensionless variables with an asterisk, $v^{*}=v(t) / u_{c i}, t^{*}=t / \tau_{c i}$, respectively.

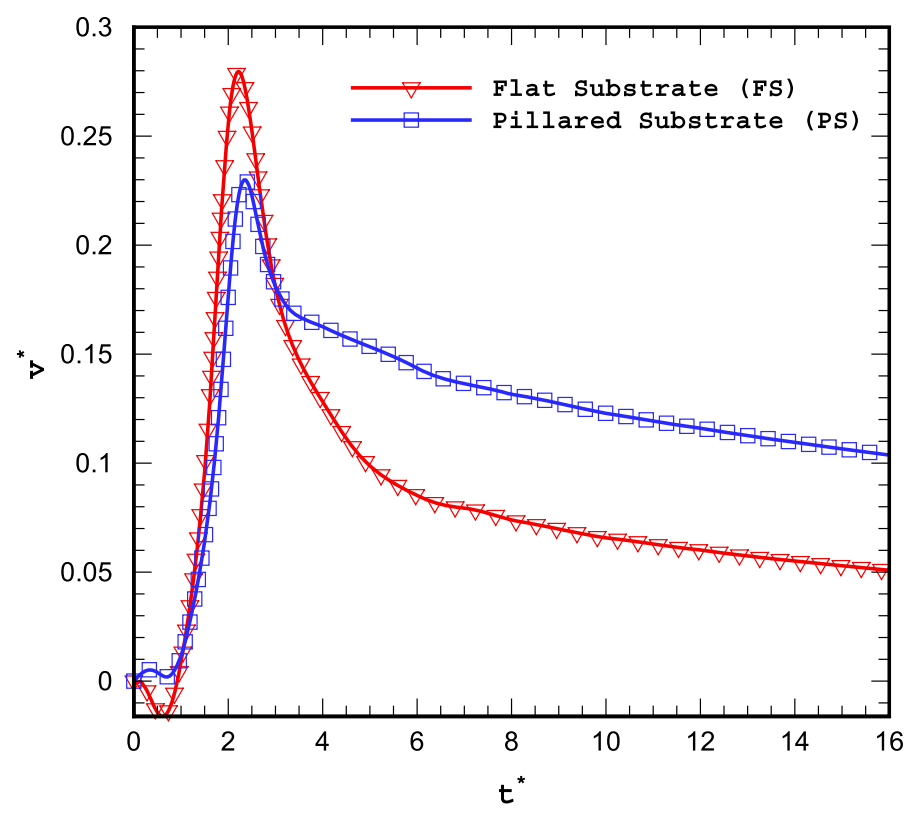

Figure 4. Comparison of time evolution of droplet velocity on a FS $\left(\theta=180^{\circ}\right)$ with that of the droplet velocity on a PS $\left(\theta=160^{\circ}\right)$ at $O h=.0375, \mu_{r}=60$, and $\rho_{r}=800$. 


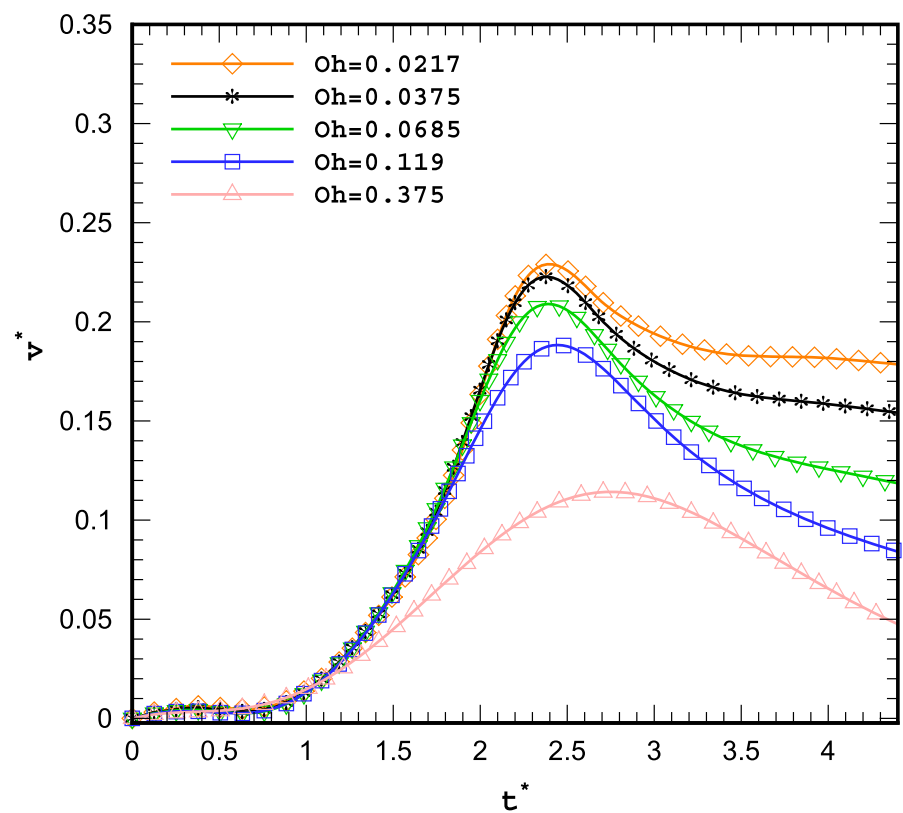

Figure 5. Time dependence of instantaneous droplet vertical velocity on PS for various Oh with $\mu_{r}=12.9$ and $\rho_{r}=50$.

released surface energy and the self-propelled jumping does not occur on the PS. It must be noted that the variation of the maximum velocity is due to the liquid viscosity, whereas the velocity curve during droplet departure is affected by the air viscosity which slows the jumping motion.

The maximum velocity of the merged droplet as a function of $\mathrm{Oh}$ prior to jumping on both FS and PS is shown in Figure 6. As explained in the description of Figure 4, the maximum velocity of the merged droplet on a FS is higher than that on a PS. However, as $\mathrm{Oh}$ increases towards unity, the maximum velocity becomes a strong function of the liquid viscosity and, consequently, there is no distinction of the maximum velocity of the droplet on both FS and PS.

Once the droplet leaves the substrate, the velocity of the merged droplet is strongly affected by the air viscosity. Figure 7 demonstrates the effect of air viscosity on the temporal evolution of instantaneous droplet vertical velocity by varying the viscosity ratio, and keeping all other parameters including the liquid viscosity and $\mathrm{Oh}$ fixed. The results in general indicate the significant effect of the air viscosity on the detachment of the merged droplet from both FS and PS. The strong viscous damping is also evident for lower viscosity ratio (i.e. $\mu_{r}=15$ ), where the merged droplet is inhibited from jumping. The results of Figure 7 show that structure of the substrate has also a strong effect on the evolution of the droplet velocity prior and after jumping. For the same air viscosity, the merged droplet can reach to the higher jumping velocity and height on PS compared to that on FS. 


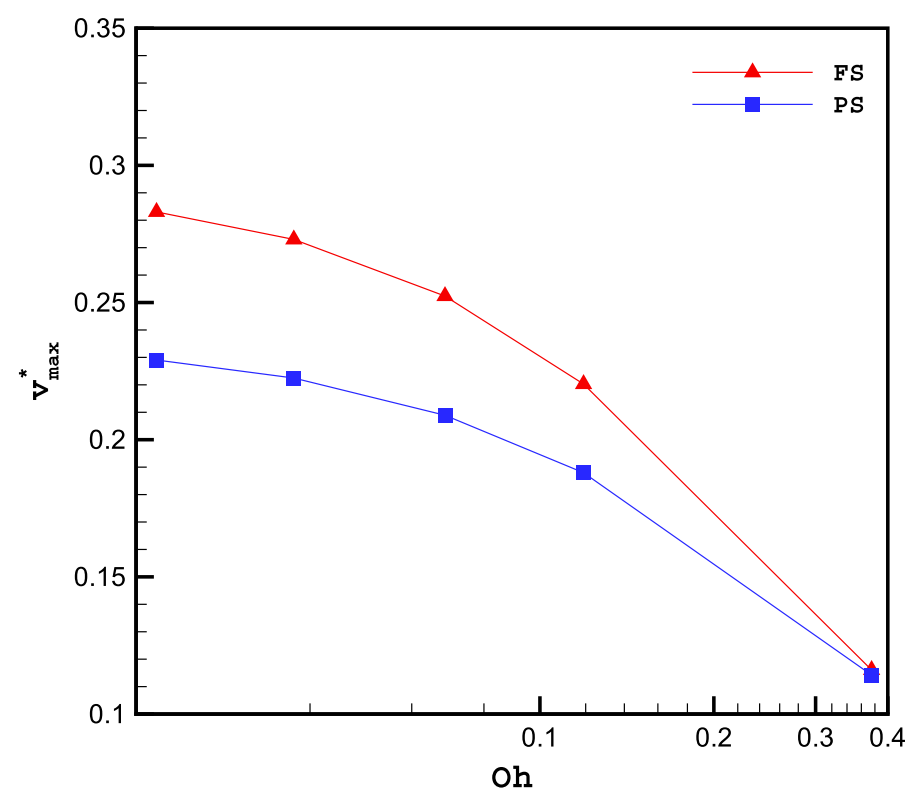

Figure 6. A comparison of the maximum instantaneous vertical velocity of the merged droplet on FS $\left(\theta=180^{\circ}\right)$ and $\mathrm{PS}\left(\theta=160^{\circ}\right)$ prior to jumping as a function of $\mathrm{Oh}$.

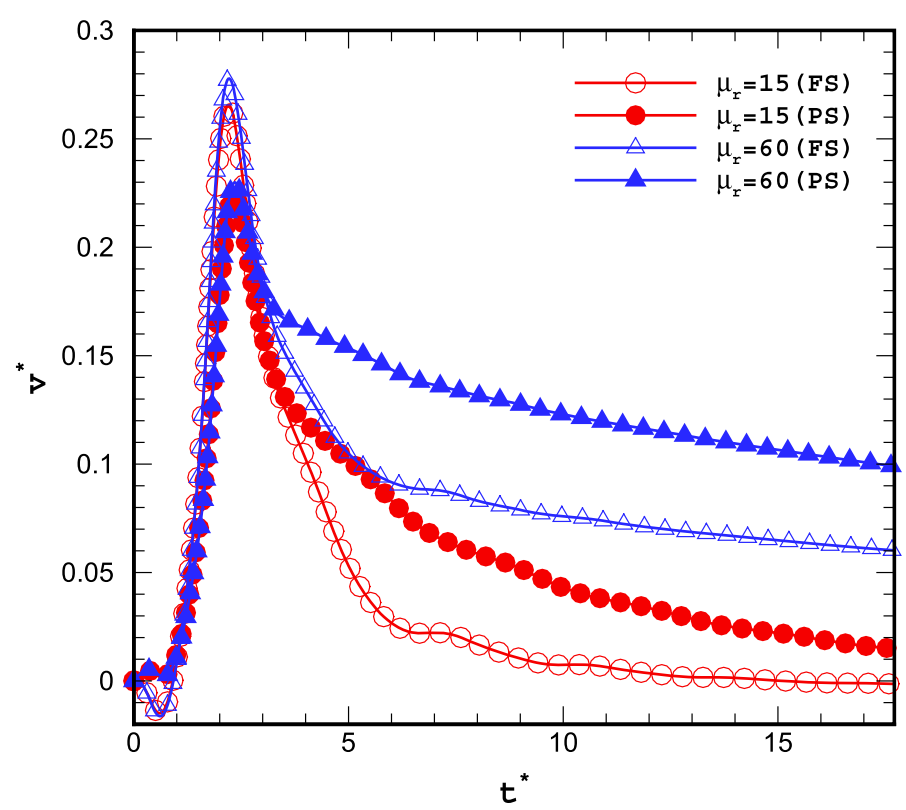

Figure 7. Time dependence of instantaneous droplet vertical velocity with $O h=.0375$ and $\rho_{r}=800$ for various air viscosities $\left(\mu_{r}=15,60\right)$.

Aside from the air viscosity, the air interia has a significant effect on the detachment of the merged droplet from the substrate at the later stages of the jumping process, which was confirmed in a recent study (Farokhirad et al., 2015) on a FS. The results of Figure 8 outlines that by decreasing the density ratio 


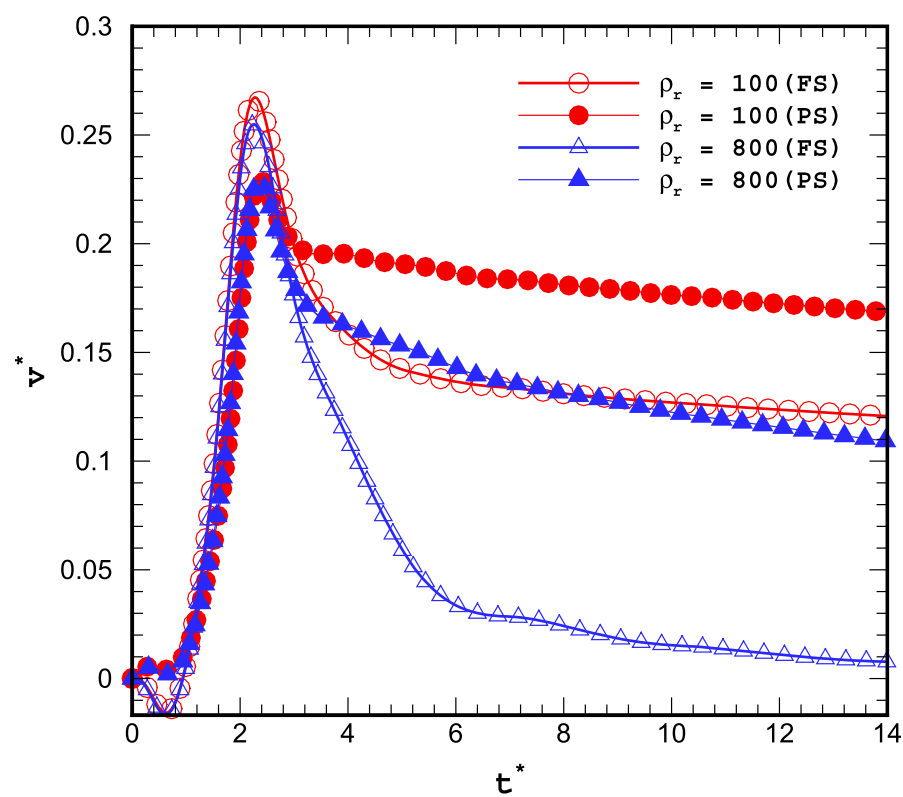

Figure 8. Time dependence of instantaneous droplet vertical velocity with $O h=.0375$ and $\mu_{r}=60$ for various air densities $\left(\rho_{r}=100\right.$, and 800$)$.

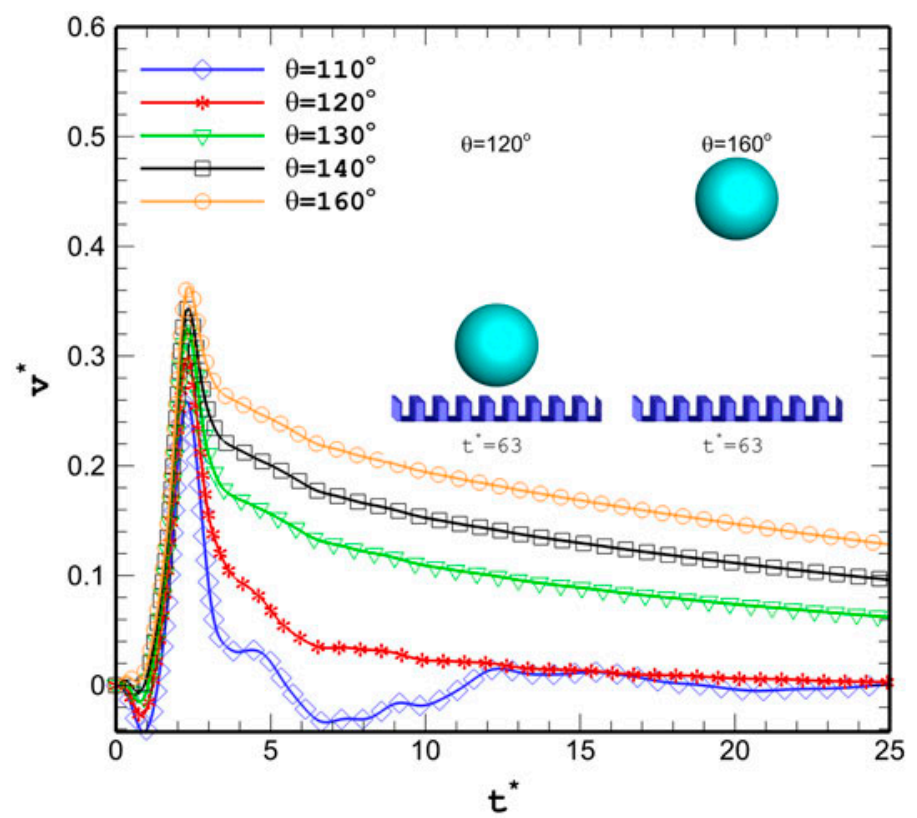

Figure 9. Effect of droplet equilibrium contact angle on its instantaneous vertical velocity for Oh $=.0375, \rho_{r}=800$ and $\mu_{r}=60$ on a PS.

(i.e. increasing the air inertia) the droplet reaches to a higher quasi-equilibrium jumping velocity and therefore to a higher vertical position, independent of the substrate structure. However, the results indicate a strong effect of the air inertia on the jumping velocity of the merged droplet on a PS, especially at the later 
$218 \Leftrightarrow$ S. FAROKHIRAD ET AL.

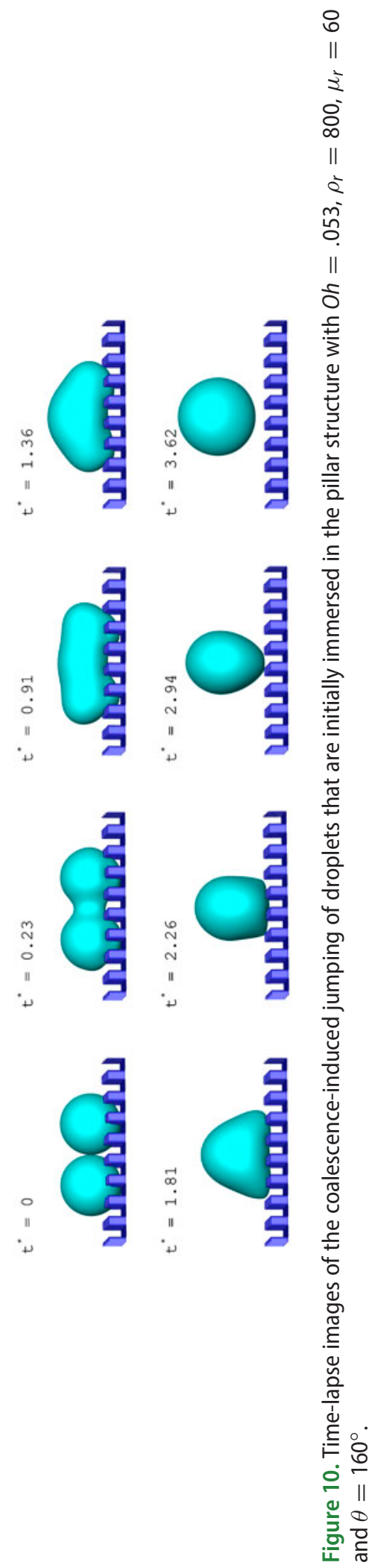




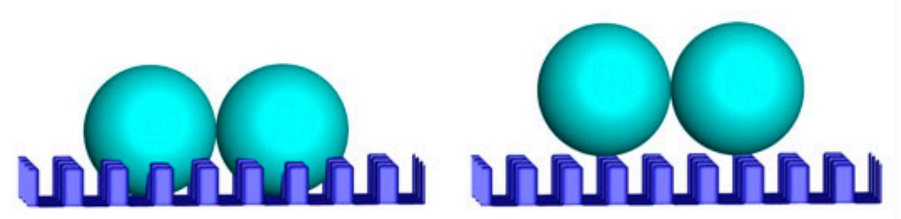

(a) $t^{*}=0$
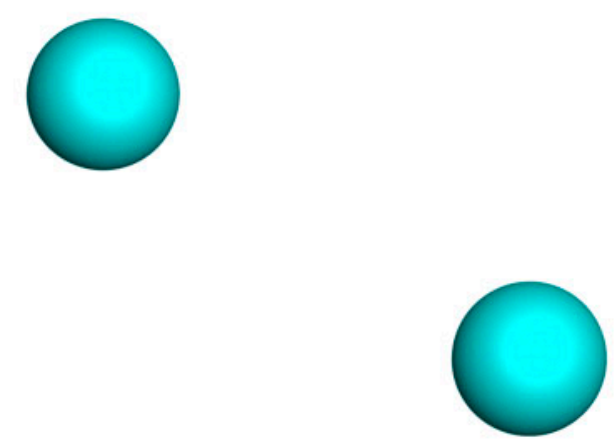

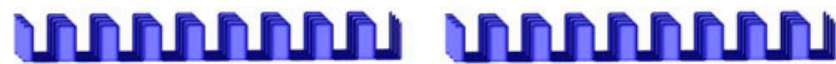

(b) $t^{*}=41$

Figure 11. A comparison of the quasi-equilibrium vertical position for the merged droplet with initial IM and SM.

stages of jumping where the droplet leaves the substrate. When a droplet leaves a FS at $\rho_{r}=100$, it reaches approximately to the same quasi-equilibrium jumping height as does a droplet on a PS at $\rho_{r}=800$.

\subsubsection{Effect of substrate wettability}

Figure 9 shows the evolution of the instantaneous droplet vertical velocity for different wettability properties of PS. Recently, it was shown that the critical contact angle where the merged droplet jumps away from an FS is independent of density ratio and is about $\theta \approx 150^{\circ}$ (Farokhirad et al., 2015). However, this criterion for a PS is reduced and the merged droplet will not jump away from a PS if the contact angle is smaller than $\theta \approx 120^{\circ}$. It is also clear that the jumping velocity, and subsequently the jumping height, becomes larger as the droplet contact angle on PS increases, as seen in Figure 9. In other words, a more superhydrophobic substrate is beneficial to induce a higher jumping velocity.

\subsection{Coalescence-induced Wenzel-Cassie wetting transition on structured superhydrophobic substrates}

In this section, we study wetting transition of coalesced droplets on a substrate with square array of pillars. In 3.1, the results are presented for droplets that are 


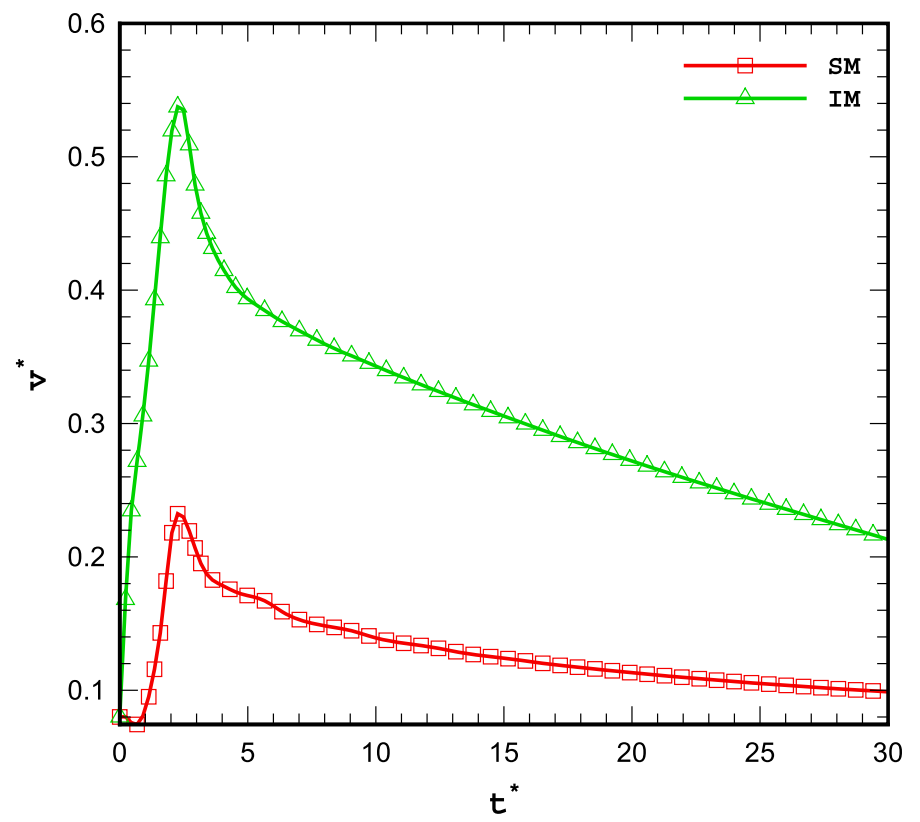

Figure 12. Time evolution of the instantaneous droplet vertical velocity during the jumping process with initial IM and SM at $O h=.053, \rho_{r}=800, \mu_{r}=60$, and $\theta=160^{\circ}$.

initially suspended on the tip of a pillar structure. To observe how the droplet's initial placement influences the jumping process, simulations with two droplets that are initially immersed within the pillars, denoted as the immersed mode (IM), are performed, as can be seen in Figure 10. The sequence of images shows how the coalesced droplets in a PS translates from the IM to the suspended mode $(\mathrm{SM})$ as the released surface energy is converted to the kinetic energy for the upward motion.

A direct comparison of the quasi-equilibrium positions of a jumping droplet in both initial IM and SM (Figure 11(a)) is given in Figure 11(b). The merged droplet with initial IM jumps higher than that with initial SM. The reason is the presence of higher initial capillary pressure in IM which is generated at the bottom contact area and makes the merged droplet jump higher from the substrate. The results of Figure 12 indicates that the entire evolution of jumping velocity with initial IM dramatically increases. The Wenzel-Cassie wetting transition in IM can be explained by the upward capillary force, generated in the gaps between nonwetting pillars. Therefore, compared to the droplet in SM, the combined effects of the capillary force and larger amount of released surface energy due to the larger contact area of droplet with PS in the IM, play roles in the enhancement of vertical jumping of the merged droplet in IM.

It must be noted that pillars on the substrate penetrate into the droplets initially in IM and occupy small volume of the droplet. To compensate for that volume and have exactly equal droplet volumes, the radius of the individual droplets in IM needs to be slightly larger than that in SM. This difference in the droplet initial 
radius depends on the pillar density. However, in this study the total reduced volume of droplets in IM is less than 1\% of the volume of droplets in SM and thus a similar initial droplet radius were considered for both modes of initial droplet placement.

\section{Conclusion}

We have adopted a recently developed three-dimensional LBM to describe the enhanced self-propelled jumping upon droplets coalescence on superhydrophobic structured substrates by changing the air inertia, the air and liquid viscosity and controlling the substrate wettability as well as the droplet initial placement. The simulation results revealed that the coalesced droplet is more likely to jump and reach a greater quasi-equilibrium height on a superhydrophobic substrate with a periodic array of pillars than on a flat substrate with contact angle of $180^{\circ}$, which is due to the reduction of adhesion between the droplet and the pillared substrate. With increasing the contact angle between the structured substrate and the droplet, the jumping height of the coalesced droplet becomes higher. The critical contact angle for coalescence-induced jumping of droplets on the structured superhydrophobic substrate is reduced compared to that for the flat substrate with a contact angle of $180^{\circ}$ and is about $120^{\circ}$. Meanwhile, the quasi-equilibrium jumping height increases correspondingly as the air inertia increases or the air viscosity decreases. For the same air inertia or viscosity, the merged droplet can reach a higher jumping velocity and height from a structured superhydrophobic substrate than a flat superhydrophobic substrate. The study further focused on the jumping comparison between droplets that are initially immersed or suspended within the pillars. The results demonstrated that as long as the size of the droplets is smaller than the capillary length, the self-propulsion mechanism can be utilised to help overcoming the energy barrier for WenzelCassie transition further. When the kinetic energy imparted by the coalescence of the immersed droplets can overcome the surface energy required to dewet the surface, a wetting transition takes place and the droplet jumps higher than when the droplet is initially suspended on the tip of the pillars.

\section{Disclosure statement}

No potential conflict of interest was reported by the authors.

\section{References}

Boreyko, J. B., \& Chen, C. H. (2009). Self-propelled dropwise condensate on super-hydrophobic surfaces. Physics Review Letters, 103, 184501. doi:10.1103/PhysRevLett.103.184501

Cahn, J. W. (1977). Critical-point wetting. The Journal of Chemical Physics, 66, 3667-3672. doi:10.1063/1.434402 
Chen, C. H., Cai, C. L., Tsai, C. L., Chen, C. L., Xiong, G., Yu, Y., \& Ren, Z. (2007). Dropwise condensation on superhydrophobic surfaces with two tier roughness. Applied Physics Letters, 90, 173108. doi:10.1063/1.2731434

Chen, X. M., Wu, J., Ma, R. Y., Hua, M., Koratkar, N., Yao, S. H., \& Wang, Z. K. (2011). Nanograssed micropyramidal architectures for continuous dropwise condensation. Advanced Functional Materials, 21, 4617-4623. doi:10.1002/adfm.201101302

Chen, X., Patel, R. S., Weibel, J. A., \& Garimella, S. V. (2016). Coalescence-induced jumping of multiple condensate droplets on hierarchical superhydrophobic surfaces. Scientific Reports, 6, 18649. doi:10.1038/srep18649

Farokhirad, S., Morris, J. F., \& Lee, T. (2015). Coalescence-induced jumping of droplet: Inertia and viscosity effects. Physics of Fluids, 27, 102102. doi:10.1063/1.4932085

Feng, J., Qin, Z. Q., \& Yao, S. H. (2012). Factors affecting the spontaneous motion of condensate drops on superhydrophobic copper surfaces. Langmuir, 28, 6067-6075. doi:10.1021/la300609f

Forsberg, P., Nikolajeff, F., \& Karlsson, M. (2011). Cassie-Wenzel and Wenzel-Cassie transitions on immersed superhydrophobic surfaces under hydrostatic pressure. Soft Matter, 7, 104-109. doi:10.1039/C0SM00595A

Kim, A., Lee, C., Kim, H., \& Kim, J. (2015). Simple approach to superhydrophobic nanostructured $\mathrm{Al}$ for practical anti-frosting application based on enhanced selfpropelled jumping droplets. ACS Applied Materials and Interfaces, 7, 7206-7213. doi:10.1021/acsami.5b00292

Lee, T. (2009). Effects of incompressibility on the elimination of parasitic currents in the lattice Boltzmann equation method for binary fluids. Computers and Mathematics with Applications, 58, 987-994. doi:10.1016/j.camwa.2009.02.017

Lee, T., \& Liu, L. (2010). Lattice Boltzmann simulations of micron-scale drop impact on dry surfaces. Journal of Computational Physics, 229, 8045-8063. doi:10.1016/j.jcp.2010.07.007

Liu, F., Ghigliotti, G., Feng, J. J., \& Chen, C. H. (2014). Numerical simulations of self-propelled jumping upon drop coalescence on non-wetting surfaces. Journal of Fluid Mechanics, 752, 39-65. doi:10.1017/jfm.2014.320

Liu, L. \& Lee, T. (2009). Wall free energy based polynomial boundary conditions for non-ideal gas lattice Boltzmann equation. International Journal of Modern Physics, 20, 1749-1768. doi:10.1142/S0129183109014710

Liu, T. Q., Sun, W., Sun, X. Y., \& Ai, H. R. (2012). Mechanism study of condensed drops jumping on super-hydrophobic surfaces. Colloids and Surfaces A, 414, 366-374. doi:10.1016/j.colsurfa.2012.08.063

Liu, X., \& Cheng, P. (2015). 3D multiphase lattice Boltzmann simulations for morphological effects on self-propelled jumping of droplets on textured superhydrophobic surfaces. International Communications in Heat and Mass Transfer, 64, 7-13. doi:10.1016/j.icheatmasstransfer.2015.03.002

Miljkovic, N., Enright, R., Nam, Y., Lopez, K., Dou, N., Sack, J., \& Wang, E. N. (2013). Jumping-droplet-enhanced condensation on scalable super-hydrophobic nanostructured surfaces. Nano Letters, 13, 179-187. doi:10.1021/nl303835d

Miljkovic, N., Enright, R., \& Wang, E. N. (2012). Effect of droplet morphology on growth dynamics and heat transfer during condensation on superhydrophobic nanostructured surfaces. NANO, 6, 1776-1785. doi:10.1021/nn205052a

Miljkovic, N., Enright, R., \& Wang, E. N. (2013). Modeling and optimization of superhydrophobic condensation. Journal of Heat Transfer, 135, 111004. doi:10.1115/1.4024597

Nam, Y., Kim, H., \& Shin, S. (2013). Energy and hydrodynamic analysis of coalescenceinduced jumping droplets. Applied Physics Letters, 103, 161601. doi:10.1063/1.4825273 
Peng, B., Wang, S., Lan, Z., Xu, W., Wen, R., \& Ma, X. (2013). Analysis of droplet jumping phenomenon with lattice Boltzmann simulation of droplet coalescence. Applied Physics Letters, 102, 151601. doi:10.1063/1.4799650

Shi, Y., Tang, G., \& Xia, H. (2015). Investigation of coalescence-induced droplet jumping on superhydrophobic surfaces and liquid condensate adhesion on slit and plain fins. International Journal of Heat and Mass Transfer, 88, 445-455. doi:10.1016/j.ijheatmasstransfer.2015.04.085

Wang, F. C., Yang, F., \& Zhao, Y. P. (2011). Size effect on the coalescence-induced selfpropelled droplet. Applied Physics Letters, 98, 053112. doi:10.1063/1.3553782

Wenzel, R. N. (1936). Resistance of solid surfaces to wetting by water. Industrial and Engineering Chemistry Research, 28, 988-994. doi:10.1021/ie50320a024

Wisdom, K. M., Watson, J. A., Liu, F., Watson, G. S., \& Chen, C. H. (2013). Selfcleaning of super-hydrophobic surfaces by self-propelled jumping condensate. Proceedings of the National Academy of Sciences of the United States of America., 110, 7992-7997. doi:10.1073/pnas.1210770110

Zhang, R., Farokhirad, S., Lee, T., \& Koplik, J. (2014). Multiscale liquid drop impact on wettable and textured surfaces. Physics of Fluids, 26, 082003. doi:10.1063/1.4892083 